\title{
SDSS J092455.87+021924.9: an Interesting Gravitationally Lensed Quasar from the Sloan Digital Sky Survey
}

\author{
Naohisa Inada, ${ }^{1}$ Robert H. Becker ${ }^{2,3}$ Scott Burles ${ }^{4}$ Francisco J. Castander ${ }^{5,6}$ Daniel \\ Eisenstein,${ }^{7}$ Patrick B. Hall,,${ }^{8,9}$ David E. Johnston, ${ }^{10}$ Bartosz Pindor, ${ }^{8}$ Gordon T. \\ Richards ${ }^{11}$ Paul L. Schechter ${ }^{4,12}$ Maki Sekiguchi, ${ }^{1}$ Richard L. White, ${ }^{13}$ J. Brinkmann, ${ }^{14}$ \\ Joshua A. Frieman, ${ }^{10,15}$ S.J. Kleinman, ${ }^{14}$ Jurek Krzesiński, ${ }^{14,16}$ Daniel C. Long, ${ }^{14}$ Eric H. \\ Neilsen, Jr.,${ }^{15}$ Peter R. Newman, ${ }^{14}$ Atsuko Nitta,${ }^{14}$ Donald P. Schneider, ${ }^{11}$ S. Snedden,${ }^{14}$ \\ and Donald G. York ${ }^{10,17}$
}

\footnotetext{
ABSTRACT

${ }^{1}$ Institute for Cosmic Ray Research, University of Tokyo, 5-1-5 Kashiwa, Kashiwa City, Chiba 277-8582, Japan.

${ }^{2}$ Physics Department, University of California, Davis, CA 95616.

${ }^{3}$ IGPP-LLNL, L-413, 7000 East Avenue, Livermore, CA 94550.

${ }^{4}$ Physics Department, Massachusetts Institute of Technology, 77 Massachusetts Avenue, Cambridge, MA 02139 .

${ }^{5}$ Department of Physics, Yale University, PO Box 208121, New Haven, CT 06520.

${ }^{6}$ Departamento de Astronomía, Universidad de Chile, Casilla 36-D, Santiago, Chile.

${ }^{7}$ Steward Observatory, University of Arizona, 933 North Cherry Avenue, Tucson, AZ 85721.

${ }^{8}$ Princeton University Observatory, Peyton Hall, Princeton, NJ 08544.

${ }^{9}$ Departamento de Astronomía y Astrofísica, Pontificia Universidad Católica de Chile, Casilla 306, Santiago 22, Chile.

${ }^{10}$ Department of Astronomy and Astrophysics, The University of Chicago, 5640 South Ellis Avenue, Chicago, IL 60637.

${ }^{11}$ Department of Astronomy and Astrophysics, The Pennsylvania State University, 525 Davey Laboratory, University Park, PA 16802.

${ }^{12}$ Institute for Advanced Study, Einstein Drive, Princeton, NJ 08540.

${ }^{13}$ Space Telescope Science Institute, 3700 San Martin Drive, Baltimore, MD 21218.

${ }^{14}$ Apache Point Observatory, P.O. Box 59, Sunspot, NM 88349.

${ }^{15}$ Fermi National Accelerator Laboratory, P.O. Box 500, Batavia, IL 60510.

${ }^{16}$ Obserwatorium Astronomiczne na Suhorze, Akademia Pedagogicazna w Krakowie, ulica Podchorążych 2, PL-30-084 Kraków, Poland.

${ }^{17}$ Enrico Fermi Institute, The University of Chicago, 5640 South Ellis Avenue, Chicago, IL 60637.
} 
We report the discovery of a new gravitationally lensed quasar from the Sloan Digital Sky Survey, SDSS J092455.87+021924.9 (SDSS J0924+0219). This object was selected from among known SDSS quasars by an algorithm that was designed to select another known SDSS lensed quasar (SDSS 1226-0006A,B). Five separate components, three of which are unresolved, are identified in photometric follow-up observations obtained with the Magellan Consortium's 6.5m Walter Baade telescope at Las Campanas Observatory. Two of the unresolved components (designated A and $\mathrm{B}$ ) are confirmed to be quasars with $z=1.524$; the velocity difference is less than $100 \mathrm{~km} \mathrm{sec}^{-1}$ according to spectra taken with the W. M. Keck Observatory's Keck II telescope on Mauna Kea. A third stellar component, designated $\mathrm{C}$, has the colors of a quasar with redshift similar to components A and B. The maximum separation of the point sources is 1 1"78. The other two sources, designated $\mathrm{G}$ and $\mathrm{D}$, are resolved. Component $\mathrm{G}$ appears to be the best candidate for the lensing galaxy. Although component $\mathrm{D}$ is near the expected position of the fourth lensed component in a four image lens system, its properties are not consistent with being the image of a quasar at $z \sim 1.5$. Nevertheless, the identical redshifts of components A and B and the presence of component $\mathrm{C}$ strongly suggest that this object is a gravitational lens. Our observations support the idea that a foreground object reddens the fourth lensed component and that another unmodeled effect (such as micro- or milli-lensing) demagnificates it, but we cannot rule out the possibility that SDSS0924+0219 is an example of the relatively rare class of "three component" lens systems.

Subject headings: gravitationally lensing — quasars: individual (SDSS J092455.87+021924.9)

\section{Introduction}

If the rate of gravitational lenses among known quasars is roughly $0.1 \%$ of all quasars (Turner, Ostriker \& Gott 1984), the expected number of new gravitationally lensed quasars in the Sloan Digital Sky Survey's (SDSS; York et al. 2000) final spectroscopic quasar sample will be approximately $10^{2}$. Furthermore, the entire SDSS photometric sample may contain an order of magnitude more lensed systems (since the photometric observations reliably probe $\sim 2$ mag fainter than the spectroscopic sample).

The SDSS is a project to conduct parallel photometric and spectroscopic surveys of 10,000 $\mathrm{deg}^{2}$ of the sky centered approximately on the North Galactic Pole, using a dedicated wide-field 2.5-m telescope at Apache Point Observatory (APO) in New Mexico, USA.

Photometric observations are done in five broad optical bands $(u, g, r, i$, and $z$, centered at 
$3561 \AA, 4676 \AA, 6176 \AA, 7494 \AA$, and $8873 \AA$ respectively (Fukugita et al. 1996; Stoughton et al. 2002). The imaging camera consists of a $5 \times 6$ array of large photometric CCDs $(2048 \times 2048$ pixels $)$ and 24 astrometric CCDs $(2048 \times 128$ pixels) (Gunn et al. 1998). The imaging data are reduced by the photometric pipeline (Lupton et al. 2001) using information from the astrometric pipeline (Pier et al. 2002) and the 0.5-m photometric calibration telescope (Hogg et al. 2001; Smith et al. 2002). Spectroscopic observations are done with a multi-fiber spectrograph covering $3800 \AA$ to $9200 \AA$ with the resolution $\mathrm{R}=1800$.

We are interested in using the SDSS to find new lensed quasars since they have proven to be useful for cosmological tests, especially for the measurement of the cosmological constant by the number counts of lensed quasars (Fukugita \& Turner 1991) as well as the measurement of the Hubble constant by observations of the time delay between multiple components (Refsdal 1964). Finding new lensed quasars in a large, homogeneous survey, such as the SDSS will contribute greatly to the statistics of lensed quasars (useful for measurements of the cosmological constant) and the determination of their time delays (useful for constraining the Hubble constant). To date the SDSS has yielded one new two-image lensed quasar, SDSS 1226-0006A,B (Inada et al. 2002) and here we report on the second lensed quasar system discovered among the SDSS quasars.

To maximize the likelihood of discovering additional lens systems, we are studying the SDSS parameters of previously known lensed quasars. In particular, we studied the parameters of SDSS J1226-0006, and using these parameters we developed an algorithm to select SDSS 1226-like objects from the SDSS database. This algorithm should be sensitive to lensed quasars with separations on the order of 1".0 to 2".5. Applying this algorithm to the approximately 10,000 SDSS quasars discovered prior to 2001 December 1, we identified five lensed quasar candidates. By definition one of them is SDSS 1226-0006. Another candidate is SDSS J0924+0219 $\left(09^{h} 24^{m} 55^{s} .87,+02^{\circ} 19^{\prime} 24^{\prime \prime} .9\right.$, J2000) which is identified as a $z=1.524$ quasar in the SDSS database. We obtained photometric follow-up observations of SDSS J0924+0219 using the Magellan Consortium's Walter Baade 6.5-m (WB6.5m) telescope at Las Campanas Observatory under good seeing conditions (0!55 0'.75). Additional spectroscopic observations of the components that were resolved by the WB6.5m images were obtained with the Keck II telescope at the W. M. Keck Observatory on Mauna Kea in < 1".0 seeing. Observations of the three other candidates from this sample have been taken with the WB6.5m telescope and indicate that these systems are also likely to be lensed quasars; these systems will be discussed in future papers.

Section 2 of this paper briefly describes the new algorithm which led to the discovery of SDSS0924+0219. Section 3 describes the follow-up observations and show the results of them. In $\S 4$, we discuss some of the interesting aspects of this system. Finally, we present 
a summary of this paper in $\S 5$.

\section{Selection Method of Finding lensed quasars from the SDSS Quasar Catalog}

We now describe the manner in which SDSS J0924+0219 was selected as a gravitational lens candidate based upon the object parameters in the SDSS object catalog (Lupton et al. 2001). First, we selected objects that were confirmed to be quasars in the SDSS spectroscopic survey (see Richards et al. 2002 for details of the SDSS quasar target selection algorithm). We rejected quasars whose redshifts are less than 0.6 since many low-redshift quasars are extended objects (Schneider et al. 2002), making it hard to distinguish them from unresolved lensed quasars. Next, we restricted our lensed quasar candidate sample using some SDSS catalogued parameters, specifically the galaxy profile fitting likelihood ${ }^{18}$. As the SDSS data are passed through the data reduction pipelines, each extended object is fitted with a set of possible galaxy profiles (Lupton et al. 2001) and labeled with the likelihood that each profile explains the data. These likelihoods are useful for searching for extended quasars, so we optimized our search criteria to use these values, based on our study of the first SDSS lensed quasar, SDSS J1226-0006.

This algorithm targets lensed quasars whose separations are approximately $1^{\prime \prime} .0 \sim 2^{\prime \prime} .5$, because we empirically confirmed that lensed quasars whose separations are less than $1^{\prime \prime} 0$ do not have large "extended" parameters in the SDSS object catalog; we cannot distinguish these small separation lensed quasars from single, unresolved quasars. For lensed quasars that have separations of more than 2.5 , each lensed component should appear as a separate entity in the SDSS catalog. As a result of applying this algorithm to the approximately 10,000 SDSS quasars (in $\sim 1,100 \mathrm{deg}^{2}$ ), we selected five lensed quasar candidates. One of these five candidates is the first SDSS lensed quasar, SDSS 1226-0006, and another of these candidates is SDSS J0924+0219. If the total lensing rate is roughly $0.1 \%$ of all quasars, then on the order of 10 lensed quasars are expected from the approximately 10,000 SDSS quasars that were known at the start of this work. About half of the lensed quasars should have 1 ". $0 \sim 2$ ".5 separations (Chiba \& Yoshii 1999); therefore, the result of this algorithm is consistent with the theoretical estimate. Whether or not this selection algorithm is the optimal way to select moderate separation lens candidates from the SDSS imaging data remains to be seen, since it could be biased towards SDSS J1226-0006-like lens systems. However, the successful discovery of two lensed quasars suggests that it is a reasonable method to use for our initial

\footnotetext{
${ }^{18}$ We mainly used two SDSS catalogued parameters, "dev_L" (de Vaucouleurs profile fitting likelihood) and "exp_L" (exponential disk fitting likelihood).
} 
lens search.

\section{Data analysis}

\subsection{Follow-up Observations}

Photometric follow-up observations of SDSS J0924+0219 were obtained using the WB6.5m telescope. The data were taken on 2001 December 15 with $u, g, r$, and $i$ filters using the Magellan Instant Camera (MagIC, a 2048×2048 CCD camera); the seeing was 0".55 0".75 FWHM. The pixel size was 0'.069. The exposure time was $300 \mathrm{sec}$ in each band. Each CCD frame was bias-subtracted and flat-field corrected.

Additional spectroscopic data were taken on 2002 January 12 with the Keck II Echellette Spectrograph and Imager (ESI; Sutin 1997), using the MIT-LL 2048×4096 CCD camera and a 175 line $\mathrm{mm}^{-1}$ grating. We used the echellette mode. The resolution of the echellette mode

of this spectrograph is $11.4 \mathrm{~km} \mathrm{sec}^{-1}$ pixel $^{-1}$. The spatial resolution scale of this spectrograph is 0 '! 153 pixel $^{-1}$. The spectral range covers $3900 \AA$ to $11,000 \AA$. The exposure time was 1200 sec. We set the slit direction so that two of the three components (components A and B, see below) were on the slit at the same time. The two components are separated by $1^{\prime \prime} .78$ and the seeing was less than 1".0 FWHM; the two components are clearly distinct in the 2-dimensional ESI image. The two spectra were extracted separately using the usual method of summing the flux in a window around each object and subtracting sky from neighboring windows on either side of the trace. The only difference from a simple single spectrum extraction was that we were careful to exclude the other object from the sky windows. There was no need (and no attempt) to fit the two spectra simultaneously because we cannot see any overlap along the slit in the 2-dimensional ESI image. However, flux of component D (see below) actually affect the component A spectrum (we set the slit width $1^{\prime \prime}$. 0 ). We could not see it in the 2-dimensional ESI image, because component D is close to the component $\mathrm{A}$, and is more than two magnitudes fainter than component $\mathrm{A}$. The estimated contaminations are $6.0 \%$ of the flux of component $\mathrm{A}$ in $g$ band $(4000 \AA \sim 5500 \AA), 6.8 \%$ in $r$ band $(5500 \AA \sim 7000 \AA)$, and $7.4 \%$ in $i$ band $(7000 \AA \sim 8500 \AA)$, respectively.

We used a single slit position that included components A and B, which puts component $\mathrm{G}$ (see below) slightly off to one side. Since it is much fainter (about 2 mag fainter than component A), it is not noticeable in the spectrum. 


\subsection{Photometry}

The SDSS images (sky and bias subtracted and flat-field corrected) are shown in all bands in Figure 1. Total magnitudes of the five components are 18.68 $\pm 0.02,18.43 \pm 0.01$, $18.34 \pm 0.01,18.09 \pm 0.01$, and $17.98 \pm 0.03$ in $u^{*}, g^{*}, r^{*}, i^{*}$, and $z^{*}$, respectively ${ }^{19}$. These errors in the magnitudes are statistical errors.

We show the full follow-up MagIC $r$ image including nearby stars (star a, star b, and star c) as well as SDSS J0924+0219 in Figure 2. The non-PSFs subtracted (hereafter "original") $u, g, r$, and $i$ images of SDSS J0924+0219 and the PSF-subtracted images of each band are shown in Figure 3. The upper panels are the original images and the lower panels are the PSF-subtracted images. We subtracted PSFs using stars from the original images in all bands. We used star a for the $u$ image, star $\mathrm{b}$ for the $r$ and the $i$ images and star $\mathrm{c}$ for the $g$ image, respectively. The "peak" flux and the center coordinates of stars a, b, and c and components A, B, and C were calculated by the single Gaussian fit (these results agree with the results obtained by the "imexamine" task in $\operatorname{IRAF}^{20}$ ). We named the three stellar components "components A-C" according to their magnitudes, the center extended object "component G" and the unknown component which remains after subtracting PSFs "component D". The flux ratios between components A and B are 0.47, 0.44, 0.43, and 0.43 in $u, g, r$, and $i$, respectively, and those between components $\mathrm{A}$ and $\mathrm{C}$ are $0.44,0.43,0.41$, and 0.40. In the original $i$ image one can see components $\mathrm{G}$ and $\mathrm{D}$, both are much more prominent in the PSF-subtracted images (except for $u$ ). We show the reduced $\chi^{2}$ from the PSF subtraction in Table 1 . The reduced $\chi^{2}$ of the $g, r$, and $i$ images of component A and component $\mathrm{C}$ are large, because component $\mathrm{A}$ has contamination from components $\mathrm{G}$ and $\mathrm{D}$, and component $\mathrm{C}$ has contamination from component $\mathrm{G}$. We cannot see components $\mathrm{D}$ and $\mathrm{G}$ in the $u$ PSF-subtracted image. This fact suggests that component $\mathrm{D}$ is not a quasar but rather a galaxy. We give our estimated magnitudes and colors of the three stellar components, component $\mathrm{G}$ and component $\mathrm{D}$ in Table 2. We used stars a, b, and c as the photometric standard stars using their SDSS catalog magnitudes and positions.

\footnotetext{
${ }^{19}$ The filters of the SDSS $2.5-\mathrm{m}$ are referred to as ugriz, while the still preliminary 2.5-m filter-based photometry will be called $u^{*} g^{*} r^{*} i^{*} z^{*}$ (Stoughton et al. 2002)

${ }^{20}$ IRAF is the Image Reduction and Analysis Facility, a general purpose software system for the reduction and analysis of astronomical data. IRAF is written and supported by the IRAF programming group at the National Optical Astronomy Observatories (NOAO) in Tucson, Arizona. NOAO is operated by the Association of Universities for Research in Astronomy (AURA), Inc. under cooperative agreement with the National Science Foundation.
} 


\subsection{Spectra}

Spectra of components A and B taken with ESI on Keck II are shown in Figure 4. Al III, Si III], C III], and Mg II emission lines are seen clearly; both components are quasars at the same redshift $(z=1.524)$. The velocity difference between the two components is less than $100 \mathrm{~km} \mathrm{sec}^{-1}$, calculated using the Mg II emission lines. The widths of the emission lines of both components are also in good agreement. We do not have a spectrum of component $\mathrm{C}$, but the colors of this component indicate that this component is also a quasar. A photometric redshift can be computed for component $\mathrm{C}$ following Richards et al. (2001), assuming an $i-z$ color of -0.025 . The resulting photometric redshift is $1.33 \pm 0.20$, which is consistent with the redshifts of components $\mathrm{A}$ and $\mathrm{C}, z=1.524$. The redshifts and the widths of the emission lines are summarized in Table 3.

\subsection{Astrometry}

We calculated the celestial coordinates of components A, B, and C based on the SDSS celestial coordinates of the three stars (star a, star b, and star c, Figure 2) common to the MagIC $r$ image and the SDSS data. The separations between components $\mathrm{A}$ and $\mathrm{B}, \mathrm{B}$ and $\mathrm{C}$, and $\mathrm{A}$ and $\mathrm{C}$ are calculated to be $1^{\prime \prime} .78 \pm 0^{\prime \prime} .04,1^{\prime \prime} .50 \pm 0^{\prime \prime} .03$, and $1^{\prime \prime} .14 \pm 00^{\prime \prime} .04$, respectively. We also calculated the celestial coordinates of component $\mathrm{G}$ and component $\mathrm{D}$ after subtracting PSFs. The results are summarized in Table 4.

\section{Discussion}

SDSS J0924+0219 is certainly a lensed quasar because of its morphology (there is a galaxy between three almost same color quasars) and the small velocity difference and the small line width differences between components A and B. However, even though there are no emission line profile differences between components $\mathrm{A}$ and $\mathrm{B}$, the ratio of the spectra of the two quasars is not constant with $\lambda$; it steadily decreases from 0.7 at $4000 \AA$ to 0.2 at $9000 \AA$ as shown in Figure 5. The flux of component D would affect the spectrum of component A, but the estimated contaminations are not large ( see $\S 3.1$ ) and it would not give a dramatically change in the spectrum of component A. The difference in the flux density ratio between the two components indicates that the two quasars might not be from the same physical source. It is possible that the slit used for the Keck spectroscopic observation was not precisely aligned along the line between components A and B; combined with differential refraction, this could have produced a spectroscopic flux density ratio different from the photometric 
flux ratio. However, this cannot be the full explanation, since it cannot explain why the spectroscopic flux ratio is not smooth (Figure 5).

Another possibility for the differences in the spectra of the two components is that continuum variations of the source quasar combined with the differential time delay causes the wavelength dependence in the flux density ratio. Continuum variations are often seen in quasars (Trévese, Kron, \& Bunone 2001), and differences in flux ratios between lensed component continua are seen in some catalogued lensed quasar systems, e.g. HE 1104-1805 (Wisotzki et al. 1995) and FBQ 0951+2635 (Schechter et al. 1998) ${ }^{21}$. We investigate whether the large differences between the photometric flux ratios (see $\S 4.1$ ) and the spectroscopic flux density ratio could be caused by the continuum variations combined with the differential time delay. We estimate the time delay between components A and B using the SIS model $\left(\right.$ Peacock 1999) ${ }^{22}$ :

$$
c \Delta t=\left(1+z_{l}\right) \frac{D_{s} D_{l}}{D_{l s}} \alpha_{E}\left(\theta_{+}-\theta_{-}\right)
$$

where $D_{s}, D_{l}, D_{l s}, \alpha_{E}$, and $z_{l}$ are the angular size distances from observer to the source quasar, from observer to the lensing galaxy, from the lensing galaxy to the source quasar, the Einstein radius in arcseconds and the redshift of the lensing galaxy, respectively. The observed separations from the center of the lensing galaxy are represented as $\theta_{+}$and $\theta_{-}$. We suppose the redshift of the lensing galaxy is 0.4 (see below), the velocity dispersion of the lensing galaxy is $230 \mathrm{~km} \mathrm{sec}^{-1}$ (see below), $H_{0}=70 \mathrm{~km} \mathrm{sec}^{-1} \mathrm{Mpc}^{-1}, \Omega_{m}=0.3$, and $\Omega_{\Lambda}=0.7$. Using these parameters, we determine a time delay of about 15 days. Quasars generally do not experience large variations, such as shown in Figure 4 (or Figure 5), in less than 15 days, therefore, there is a significant possibility that other additional phenomena cause the differences between the two components of SDSS J0924+0219.

One such effect might be microlensing. We cannot see clear evidence of microlensing in both spectra (Figure 4), but microlensing should be universal in quadruple lens (Witt, Mao, \& Schechter 1995), therefore, it is natural that microlensing events happen to both components or to one of the two components and cause the difference between the two components (if we suppose the same situation of Q2237+0305 (Walsh, Carswell, \& Weymann 1979), the microlensing optical depth (Schmidt, Webster, \& Lewis 1998) supports this fact). Furthermore, if there is another object which darkens component D or component D is a second lensing galaxy (see below discussion), it might have an effect on the observed spectrum

\footnotetext{
${ }^{21}$ Differential extinctions might also cause the differences of the flux ratios between the components of these two known lenses (Falco et al. 1999).

${ }^{22}$ We applied our SIS model to B $1600+434$ whose time delay is known to be about 50 days (Koopmans et al. 2000). Our calculation gave 35 days for this lensed quasar — in rough agreement with the observation.
} 
of component A, i.e. the reddening of component A could be larger than that of component B. However, this extinction should be time invariant (effectively), and therefore, we should see the same extinction in the the photometric flux ratios, but we cannot see it. Confirming spectra and direct imaging are needed to determine whether the differences between the spectroscopic and direct imaging flux ratios are real or an observational artifact.

Although the SIS model with an external shear (Kochanek 1991) predicts the existence of a fourth lensed component whose amplification is as bright as the brightest of the other three lensed components, there are no stellar components except components A, B, and C in all the images (Figure 3). Using the positions of components $\mathrm{A}-\mathrm{C}$ and of lensing galaxy $\mathrm{G}$ (which of course might not be the only lensing galaxy) we can fit the SIS model with an external shear, with a projected potential:

$$
\psi(r, \phi)=\alpha_{E} r+\frac{\gamma}{2} r^{2} \cos \left(2 \phi-2 \phi_{\gamma}\right)
$$

where $\alpha_{E}$ is the Einstein radius of the SIS model in arcseconds, $r$ and $\phi$ are the radial and the angular parts, respectively, of the angular position on the sky, and $\phi_{\gamma}$ is the position angle of the shear, measured East of North. Fitting this model to the positions (but not the fluxes) of components $\mathrm{A}-\mathrm{C}$, we get $\alpha_{E}=0.850, \gamma=0.065$, and $\phi_{\gamma}=173.65^{\circ}$, with a source position $(\Delta$ R.A., $\Delta$ Dec. $)=\left(0.025,-0{ }^{\prime \prime} .04\right)$ relative to component $\mathrm{G}$. This value of $\alpha_{E}$ corresponds to a velocity dispersion $230 \mathrm{~km} \mathrm{sec}^{-1}$ with the estimated redshift of the lensing galaxy (see final paragraph of this section). The observed positions of components $\mathrm{A}-\mathrm{C}$, the observed position of component $\mathrm{D}$ and the predicted positions of the lensed components are given in Table 5 below. With six constraints and five free parameters it is no surprise that we obtain small residuals $(<0$ '.04) from the observed positions of components A-C. Although the flux ratios of observed components were not taken as constraints, the predicted flux ratios between components $\mathrm{A}$ and $\mathrm{B}$ and between components $\mathrm{A}$ and $\mathrm{C}$ are in agreement with the observations. The predicted position of the fourth lensed component (hereafter, component $\mathrm{D}^{\prime}$ ) is shown in Figure 6a and Figure 6b. Figure 6a is the image plane of this model, and Figure $6 \mathrm{~b}$ is the enlarged part of the $i$ subtracted image of Figure 3 with the measured positions of components $\mathrm{A}-\mathrm{C}$, component $\mathrm{D}$ and the predicted position of component $\mathrm{D}^{\prime}$. Component $\mathrm{D}$ is separated by only about $0^{\prime \prime} .15$ from the predicted position of component $\mathrm{D}^{\prime}$ (the filled circle on Figure 6a or the open circle on Figure 6b). Also, the predicted position of component $\mathrm{D}^{\prime}$ is within the region occupied by component $\mathrm{D}$ (Figure $6 \mathrm{~b}$ ).

These results suggest that it is possible that either 1) component D really is the "missing" fourth lensed component, or 2) that component D is a object which is mixed component $\mathrm{D}^{\prime}$ (predicted fourth lensed component) with a foreground object that is obscuring the light from component $\mathrm{D}^{\prime}$. According to Schechter \& Wambsganss 2002, microlensing causes demagnificating (or vanishing) the fourth lensed image at "saddlepoint". The 
difference between the expected magnitude and the observed magnitude is large $(\Delta m=$ $m_{\text {expected }}-m_{\text {observed }}=-2.7$ ), but this marginally consistent with Figure 3 of Schechter \& Wambsganss 2002. Futhermore, some literatures reported that milli-lensing and/or microlensing produce anomalous flux ratios of four-image gravitationally lensed systems (Subramanian, Chitre, \& Narasimha 1985, Metcalf \& Madau 2001, Keeton 2003, and Kochanek \& Dalal 2003). The second case is also likely. If there are a foreground dusty galaxy superposed upon component $\mathrm{D}^{\prime}$, it could be obscuring and reddening the light from component $\mathrm{D}^{\prime}$. This might also explain why component $\mathrm{D}$ is bluer than component $\mathrm{G}$; component $\mathrm{D}$ may have some contribution from component $\mathrm{D}^{\prime}$, which is expected to be relatively blue. The reduced $\chi^{2}$ of component D from the PSF subtraction in the $i$ image is 20.91 and it is comparable with the other stellar component (Table 1), therefore, there is a possibility that component $\mathrm{D}$ includes a stellar component. In addition to reddening by the foreground object, the demagnifying effect of microlensing might also darken component $\mathrm{D}^{\prime}$, and therefore, component $\mathrm{D}$ might be much fainter than component $\mathrm{D}^{\prime}$.

Two additional minor possibilities are that component $\mathrm{D}$ is a second lensing galaxy and contributes significantly to the lensing potential, which would change the lensing model such that the predicted position of component $\mathrm{D}^{\prime}$ is incorrect, and that this lensing system has a very interesting lensing potential which produces only three lensed components. According to Keeton, Kochanek, \& Seljak (1997); Kassiola \& Kovner (1993); and Wallington \& Narayan (1993), there are some cases that non-singular lensing potentials with large shears, large ellipticities, or large core radii produce only three lensed component. However, SDSS J0924+0219 cannot be explained by these standard lensing models, such as non-singular lensing potentials with large shears because components of a "standard" three component lensing system are expected to be on the same side of the lensing galaxy in the non-singular lens models (Kassiola \& Kovner 1993; Wallington \& Narayan 1993) while the three components of SDSS J0924+0219 are not on the same side of the presumed lensing galaxy (component G). Spectroscopy of image D is just one way for the question to be resolved. Higher resolution imaging, say with HST, may resolve it without any need for spectra.

According to Fukugita, Shimasaku, \& Ichikawa (1995), a typical elliptical galaxy at $0.2<z<0.5$ has $1.3<g-r<1.8$ and $0.5<r-i<1.0$, which are close to the estimated colors of component $\mathrm{G}$ (see Table 2). The estimated colors and the spherical appearance of component $\mathrm{G}$, therefore, indicate that it may be an elliptical galaxy at $0.2<z<0.5$. This would be the primary lensing galaxy of this lensing system. If the redshift of the lensing galaxy is 0.4 , the 1".8 separation of components A and B requires the velocity dispersion of the lensing galaxy to be about $230 \mathrm{~km} \mathrm{sec}^{-1}$ in the SIS model, while the Faber-Jackson law predicts $225 \mathrm{~km} \mathrm{sec}^{-1}$ from the $i$ magnitude of this galaxy $(i=20.8)$. Here we assume that $M_{i}^{*}=-21.3, \sigma^{*}=225 \mathrm{~km} \mathrm{sec}^{-1}$ (Blanton et al. 1999 and Kochanek 1996), $\Omega_{\mathrm{M}}=0.3$, 
$\Omega_{\Lambda}=0.7, H_{0}=70 \mathrm{~km} \mathrm{sec}^{-1} \mathrm{Mpc}^{-1}$, and the $K$-corrections of $i$ are 0.2 for a $z=0.2$ elliptical galaxy and 0.4 for a $z=0.4$ (Inada 2001). This result favors a redshift of 0.4 for the lensing galaxy, and a velocity dispersion of about $230 \mathrm{~km} \mathrm{sec}^{-1}$.

\section{Summary and Conclusion}

Using a selection algorithm tuned to recover a previously discovered lensed quasar (SDSS 1226-0006), we have identified several additional lensed quasar candidates from the SDSS data. We confirmed that one of them, SDSS J0924+0219, is a lensed quasar with follow-up observations using the Walter Baade 6.5-m and the Keck II telescopes. The redshift of the source quasar is $z=1.524$. The maximum separation is 1".78. The velocity difference between component A and component B is very small, less than $100 \mathrm{~km} \mathrm{sec}^{-1}$. We can directly see the lensing galaxy in the original MagIC $i$ image, and we can see it more clearly in the PSF-subtracted images. The estimated colors and the magnitudes of the lensing galaxy are consistent with those of a typical elliptical galaxy at $z=0.4$ with a velocity dispersion of $230 \mathrm{~km} \mathrm{sec}^{-1}$. We can see only a faint red component near the predicted position of the fourth lensed image (using the SIS model with an external shear). We consider that this faint red component is a fourth lensed component darkened and reddened by a foreground objects and microlensing. To settle the issue of what causes the lack of the fourth quasar component, we need to obtain deeper and higher resolution images and fainter spectroscopy of component D.

Funding for the creation and distribution of the SDSS Archive has been provided by the Alfred P. Sloan Foundation, the Participating Institutions, the National Aeronautics and Space Administration, the National Science Foundation, the U.S. Department of Energy, the Japanese Monbukagakusho, and the Max Planck Society. The SDSS Web site is http://www.sdss.org/. The SDSS is managed by the Astrophysical Research Consortium (ARC) for the Participating Institutions. The Participating Institutions are The University of Chicago, Fermilab, the Institute for Advanced Study, the Japan Participation Group, The Johns Hopkins University, Los Alamos National Laboratory, the Max-Planck-Institute for Astronomy (MPIA), the Max-Planck-Institute for Astrophysics (MPA), New Mexico State University, Princeton University, the United States Naval Observatory, and the University of Washington. G. T. R. and D. P. S. acknowledge support from National Science Foundation grant AST99-00703 and D. J. E. acknowledges support from National Science Foundation grant AST00-98577 and an Alfred P. Sloan Research Fellowship. Finally, we thank the staffs of the Keck and Las Campanas Observatories for their excellent assistance. 


\section{REFERENCES}

Blanton. M. R., et al. 2001, AJ, 121, 2358

Chiba, M. \& Yoshii, Y. 1999, ApJ, 510, 42

Falco, E. E., et al. 1999, ApJ, 523, 617

Fukugita, M., Ichikawa, T., Gunn, J. E., Doi, M., Shimasaku, K., \& Schneider, D. P. 1996, AJ, 111, 1748

Fukugita, M., Shimasaku, K., \& Ichikawa, T. 1995, PASP, 107, 945

Fukugita, M. \& Turner, E. L. 1991, MNRAS, 253, 99

Gunn, J. E., et al. 1998, AJ, 116, 3040

Hogg, D. W., Finkbeiner, D. P., Schlegel,D. J., \& Gunn, J. E. 2001, AJ, 122, 2129

Inada, N. 2001, Master's thesis of University of Tokyo, Department of Physics.

Inada, N., et al. 2003, in preparation

Kassiola, A. \& Kovner, I. 1993, ApJ, 417, 450

Keeton, C. R., 2003, ApJ, 584, 664

Keeton, C. R., Kochanek, C. S., \& Seljak, U. 1997, ApJ, 482, 604

Kochanek, C. S., \& Dalal, N. 2003, ApJ, submitted (astro-ph/0302036)

Kochanek, C. S., 1996, ApJ, 466, 638

Kochanek, C. S., 1991, ApJ, 373, 354

Koopmans, L. V. E., de Bruyn, A. G., Xanthopoulos, E., \& Fassnacht, C. D. 2000, A\&A, 356,391

Lupton, R. H., Gunn, J. E., Ivezić, Ź., Knapp, G. R., Kent, S., \& Yasuda, N. 2001, ADASS X, 2001adass-10, 269

Metcalf, B. R., Madau, P, 2001, ApJ, 563, 9

Peacock, J. A., 1999, Cambridge University Press, "Cosmological Physics" 
Pier, J. R., Munn, J. A., Hindsley, R. B., Hennessy, G. S., Kent, S. M., Lupton, R. H., \& Ivezić, Ź. 2002, ApJ, submitted

Refsdal, S. 1964, MNRAS, 128, 307

Richards, G. T., et al. 2002, AJ, 123, 2945

Richards, G. T., et al. 2001, AJ, 122, 1151

Schechter, P. L., Gregg, M. D., Becker, R. H., Helfand, D. J., \& White, R. L. 1998, AJ, 115, 1371

Schechter, L. P. \& Wambsganss. 2002, ApJ, submitted (astro-ph/0204425)

Schneider, D. P., et al. 2002, AJ, 123, 567

Smith, A., et al. 2002, AJ, 123, 2121

Stoughton, C., et al. 2002, AJ, 123, 485

Subramanian, K., Chitre, S. M., \& Narasimha, D. 1985, ApJ, 289, 37

Sutin, B. M. 1997, Proc. SIPE, 2871, 1116

Wallington, S. \& Narayan, R. 1993, ApJ, 403, 517

Trévese, D., Kron, R. G., \& Bunone, A. 2001, ApJ, 551, 103

Turner, E. L., Ostriker, J. P., \& Gott, J. R., III 1984, ApJ, 284, 1

Wisotzki, L., Koehler, T., Ikonomou, M., \& Reimers, D. 1995, A\&A, 297, L59

Witt, H. J., Mao, S., \& Schechter, L. P. 1995, ApJ, 443, 18

York, D. G., et al. 2000, AJ, 120, 1579 


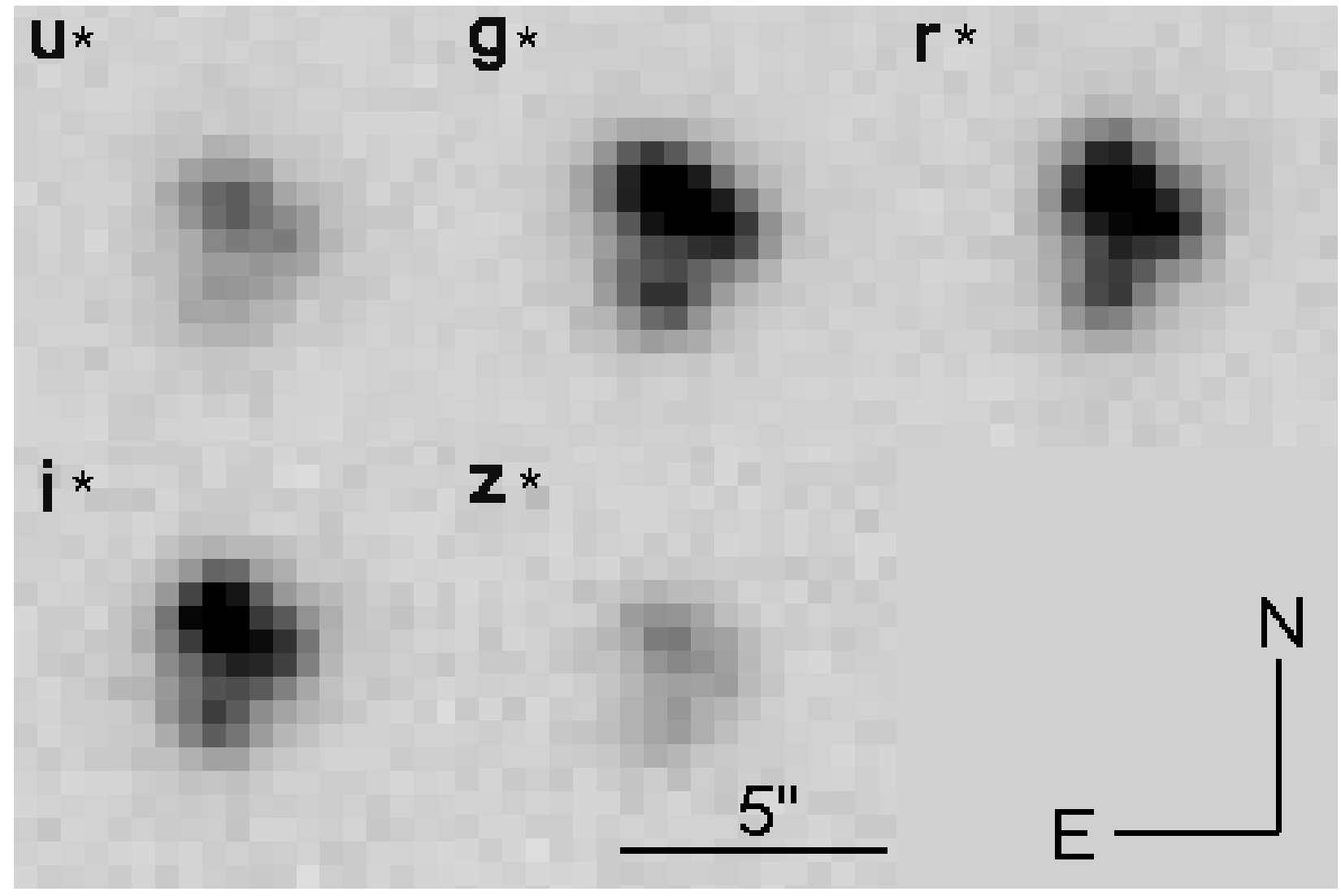

Fig. 1. - The SDSS image of SDSS J0924+0219 in all bands. The pixel size is $00^{\prime} 396$. The seeing of this field was 1".4. Total magnitudes are 18.68, 18.43, 18.34, 18.09, and 17.98 in $u^{*}$, $g^{*}, r^{*}, i^{*}$, and $z^{*}$, respectively. 


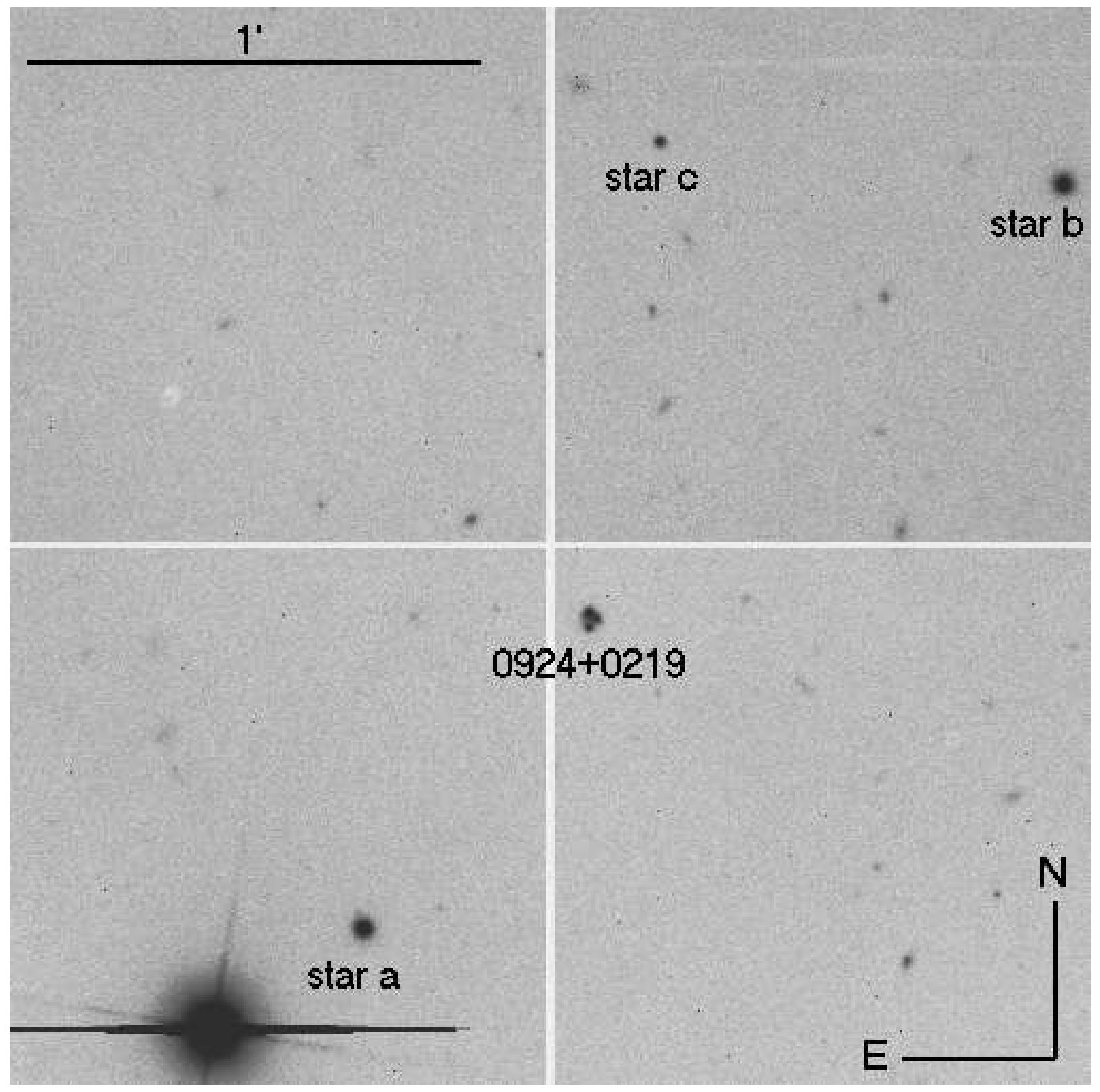

Fig. 2.- The MagIC $r$ image of SDSS J0924+0219 with nearby stars. This CCD has four readout quadrants, which appear as four separate images in this picture. 


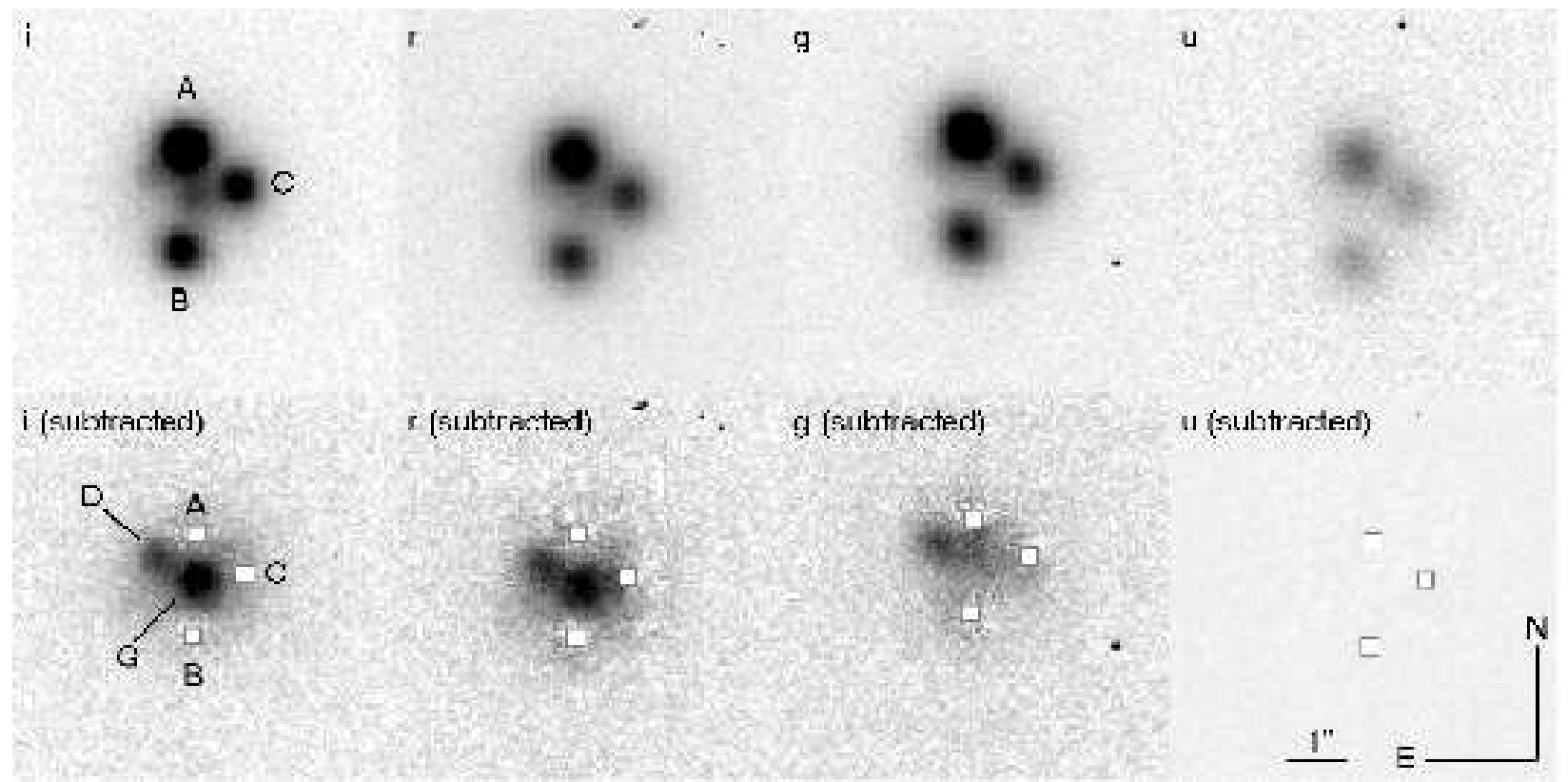

Fig. 3.- The upper panels are the MagIC images of SDSS J0924+0219 and the lower panels are the PSF-subtracted images. The open squares in the right panels represent the measured centers of components A-C. We can clearly see the three stellar components $(\mathrm{A}-\mathrm{C})$ in all original images. We can also see the central extended object $(\mathrm{G})$ and component $\mathrm{D}$ at the upper left of component $\mathrm{G}$ in the $i$ original image (we can see them more clearly in the PSF-subtracted image). We can see the central extended object $(G)$ and the unknown fifth component (D) at the upper left of component $\mathrm{G}$ in the $i, r$ and $g$ PSF-subtracted image. However, we cannot see any other components in the PSF-subtracted image. The $i, r$ and $g$ magnitudes of component D are estimated to be about 21.6, 21.8 and 22.5, respectively, and those of component $\mathrm{G}$ are estimated to be about 20.8, 21.3, and 22.7, respectively. The pixel size of these images is $00^{\prime \prime} 069$, and the seeing was less than 0.75 . The exposure time was 300 seconds. 


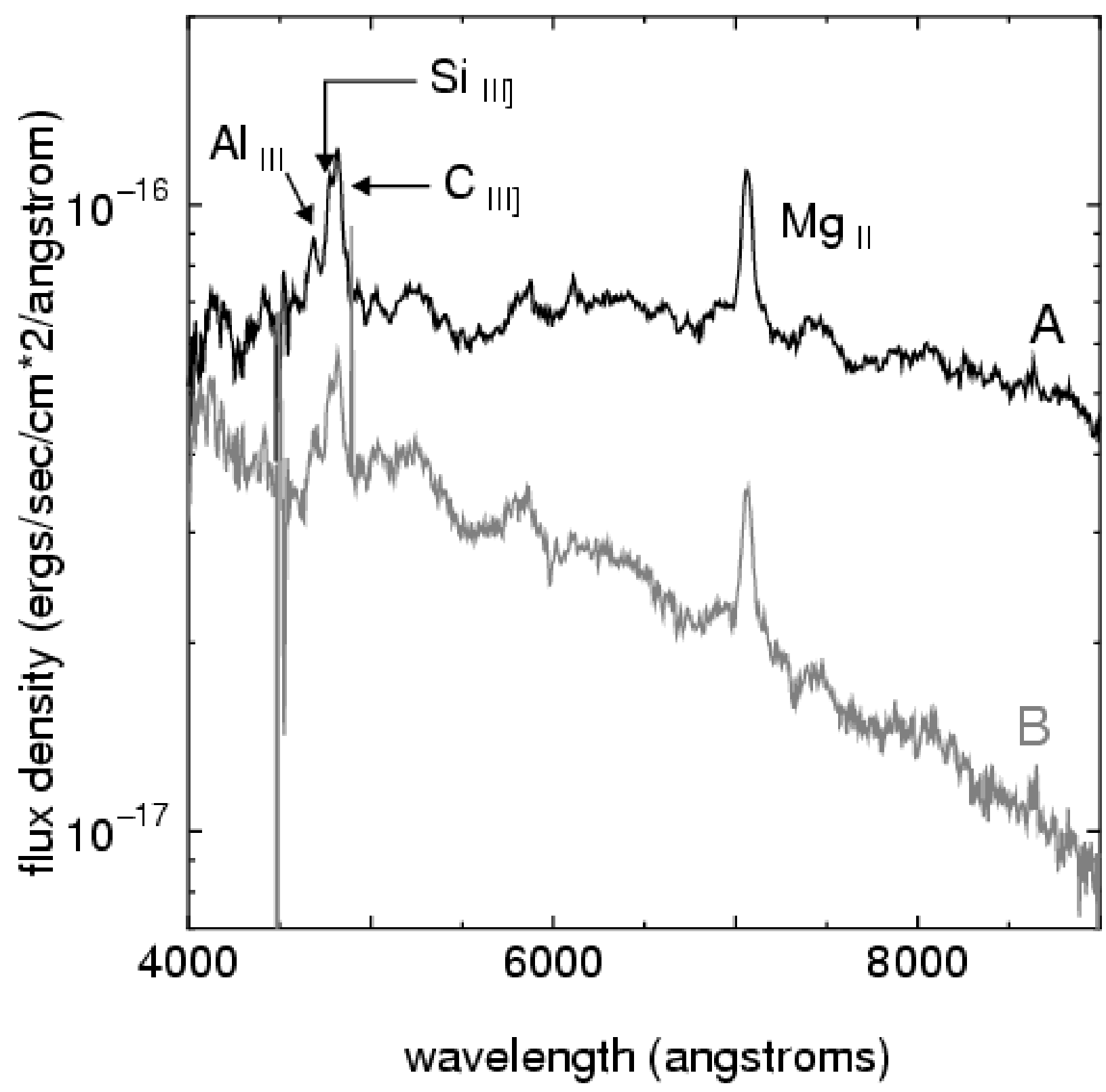

Fig. 4.- Spectra of SDSS J0924+0219 components A and B taken with ESI on Keck II. Both components have Al III, Si III], C III], and Mg II emission line at $z=1.524$. There are some bad columns around $4500 \AA$ and $4900 \AA$. 


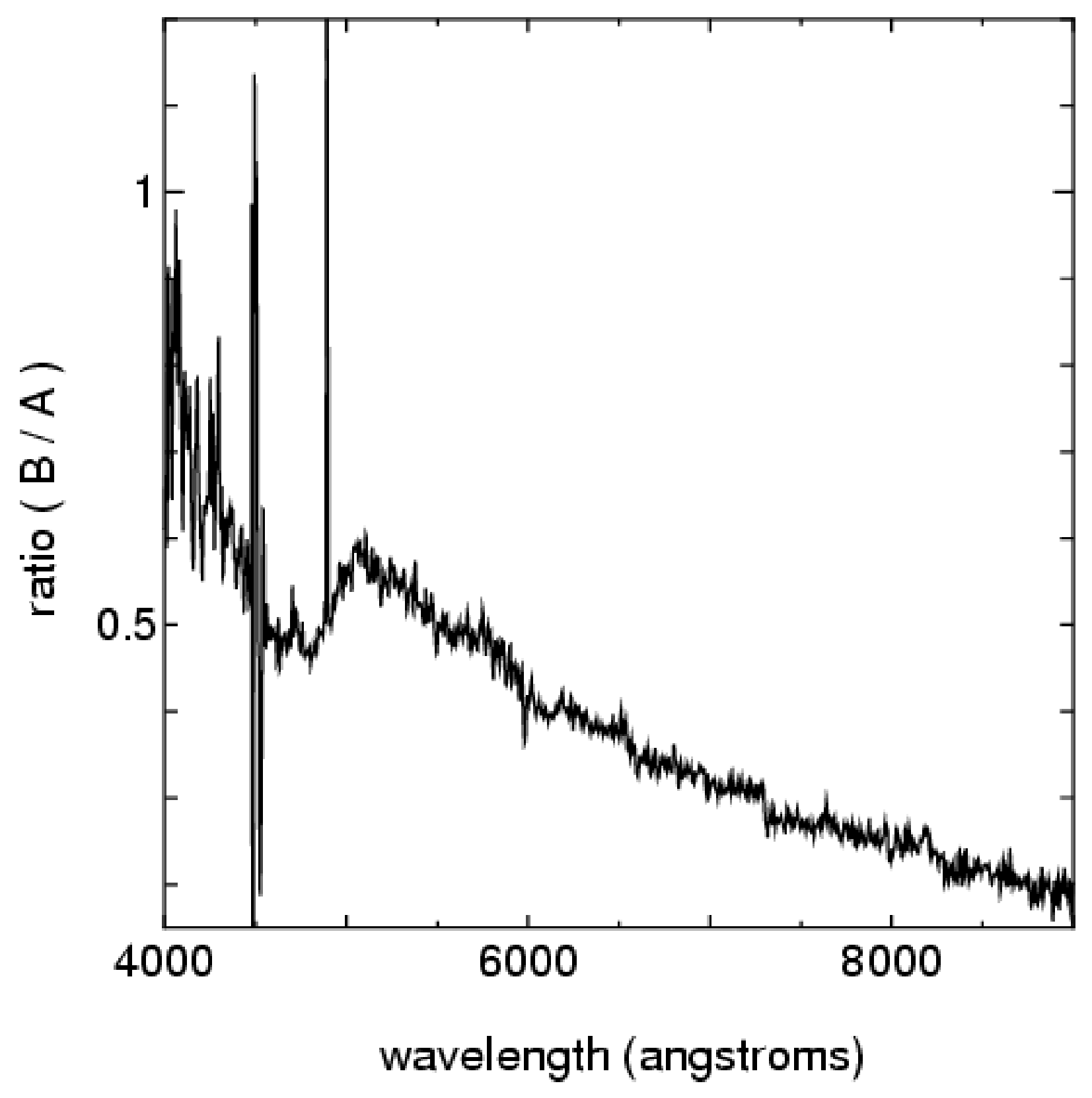

Fig. 5.- The flux ratio between the two components (A and B) of SDSS J0924+0219. Although the flux ratio is not constant, it could be explained by the continuum variations combined with the differential time delay of each component and/or microlensing. There are some bad columns around $4500 \AA$ and $4900 \AA$. 

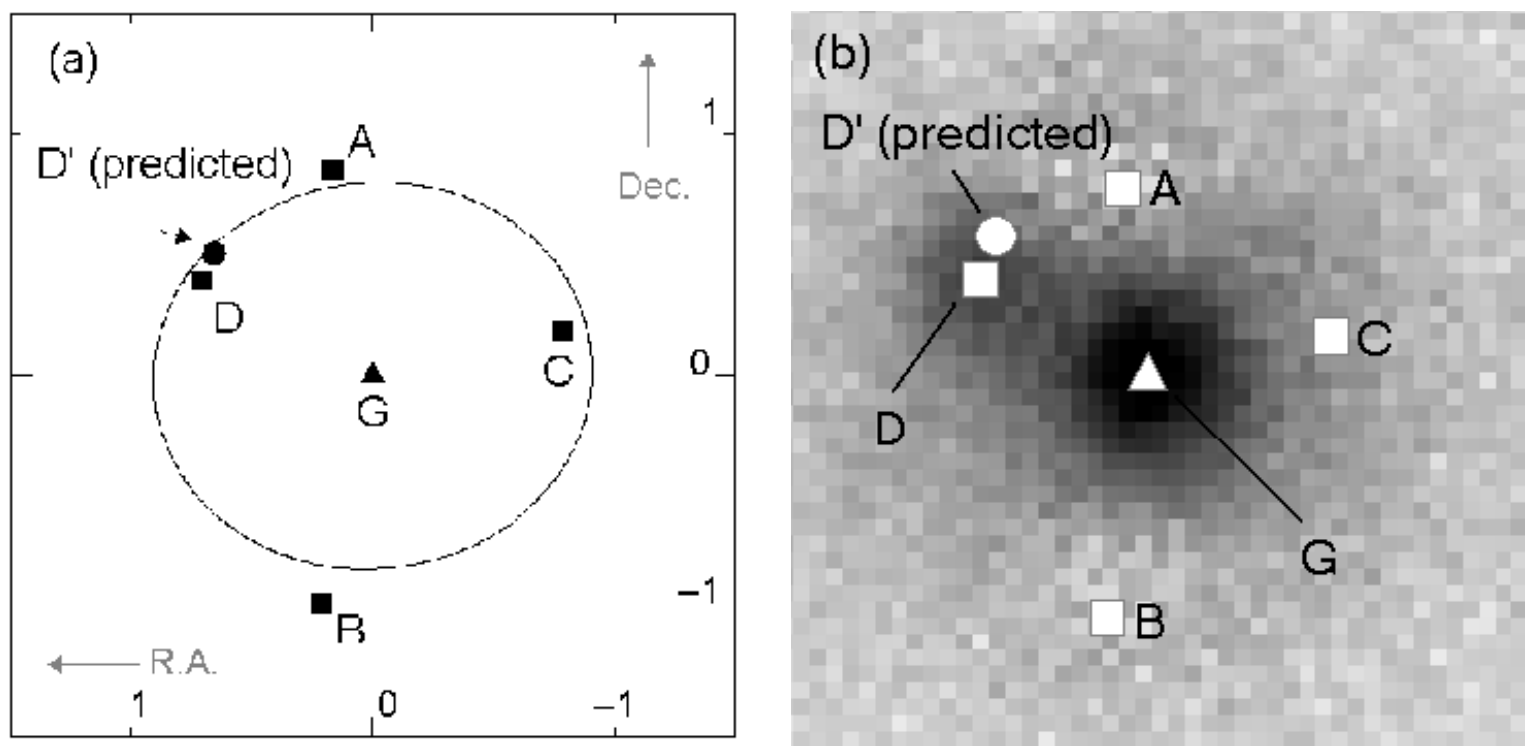

Fig. 6.- Positioning of the components relative to component G, including the predicted location of a fourth lensed component, as discussed in the text. (Left) The image plane of the model, the filled circle represents the predicted position of component $\mathrm{D}^{\prime}$ and the filled squares represent the measured position of components $\mathrm{A}-\mathrm{C}$ and component $\mathrm{D}$. The filled triangle represents the measured position of component G. (Right) An enlarged part of the $i$ subtracted image of Figure 3 (at the same scale as the left panel). The white symbols in the right panel correspond to the filled symbols in the left hand image. The predicted position of component $\mathrm{D}^{\prime}$ was calculated using the SIS model with an external shear restricted only by the positions of the other three lensed components. The observed position of component $\mathrm{D}$ is separated by only about $0^{\prime \prime} 15$ from the predicted position of component $\mathrm{D}^{\prime}$, and the predicted position of component $\mathrm{D}^{\prime}$ is within the contours of component D. Component D, however, is too red to be a quasar (Table 2). Both panels are same size, $3^{\prime \prime} \times 3^{\prime \prime}$ and are centered on the position of component $\mathrm{G}$. 
Table 1. REDUCED $\chi^{2}$ FROM PSF SUBTRACTION

\begin{tabular}{ccrcc}
\hline \hline Object & $u$ & \multicolumn{1}{c}{$g$} & \multicolumn{1}{c}{$r$} & $i$ \\
\hline Component A & 3.52 & 43.41 & 34.2 & 64.1 \\
Component B & 2.32 & 5.45 & 5.10 & 19.0 \\
Component C & 6.11 & 12.94 & 32.5 & 39.0 \\
\hline
\end{tabular}


Table 2. PHOTOMETRY FOR SDSS J0924+0219 AND NEARBY STARS

\begin{tabular}{|c|c|c|c|c|c|c|c|}
\hline Object & $u^{\mathrm{a}}$ & $g^{\mathrm{a}}$ & $r^{\mathrm{a}}$ & $i^{\mathrm{a}}$ & $u-g$ & $g-r$ & $r-i$ \\
\hline Component $\mathrm{A}^{\mathrm{b}}$ & $19.66 \pm 0.02$ & $19.46 \pm 0.01$ & $18.97 \pm 0.02$ & $18.87 \pm 0.02$ & 0.20 & 0.49 & 0.10 \\
\hline Component $\mathrm{B}^{\mathrm{b}}$ & $20.49 \pm 0.05$ & $20.34 \pm 0.04$ & $19.89 \pm 0.04$ & $19.79 \pm 0.03$ & 0.15 & 0.45 & 0.10 \\
\hline Component $\mathrm{C}^{\mathrm{b}}$ & $20.55 \pm 0.05$ & $20.38 \pm 0.05$ & $19.94 \pm 0.04$ & $19.91 \pm 0.03$ & 0.17 & 0.44 & 0.03 \\
\hline Component $\mathrm{D}^{\mathrm{b}, \mathrm{c}}$ & $c>22.30^{\mathrm{d}}$ & $22.45 \pm 0.12$ & $21.82 \pm 0.06$ & $21.61 \pm 0.05$ & $\ldots$ & 0.63 & 0.21 \\
\hline Component $\mathrm{G}^{\mathrm{b}, \mathrm{c}}$ & $c>22.30^{\mathrm{d}}$ & $22.73 \pm 0.13$ & $21.25 \pm 0.05$ & $20.78 \pm 0.05$ & $\ldots$ & 1.48 & 0.47 \\
\hline Star a & $20.80 \pm 0.01$ & $18.06 \pm 0.01$ & $16.68 \pm 0.02$ & $16.03 \pm 0.01$ & 2.74 & 1.38 & 0.65 \\
\hline Star b & $20.72 \pm 0.09$ & $18.13 \pm 0.01$ & $16.72 \pm 0.02$ & $15.68 \pm 0.01$ & 2.59 & 1.41 & 1.04 \\
\hline Star c & $23.15 \pm 0.06$ & $21.01 \pm 0.04$ & $19.47 \pm 0.02$ & $18.57 \pm 0.02$ & 2.14 & 1.54 & 0.90 \\
\hline
\end{tabular}

${ }^{a}$ We used stars $\mathrm{a}, \mathrm{b}$ and $\mathrm{c}$ as photometric standard stars. The error bars of components A, B, $\mathrm{C}, \mathrm{D}$ and $\mathrm{G}$ do not include the photometric uncertainties of these standards. The magnitude of component $\mathrm{D}$ is estimated using the flux integrated in the $20 \mathrm{kpc}$ (corresponding to 50 pixels (about 3'.7) for $z=0.4$, supposing $\Omega=0.3, \Omega_{\Lambda}=0.7$, and $H_{0}=70 \mathrm{~km} \mathrm{sec}^{-1} \mathrm{Mpc}^{-1}$ ) diameter regions centered on each component location, after subtracting the other components.

${ }^{\mathrm{b}} \mathrm{A}, \mathrm{B}, \mathrm{C}, \mathrm{G}$, and D are denoted in Figure 3.

${ }^{\mathrm{c}}$ Components $\mathrm{G}$ and $\mathrm{D}$ are not observed in the PSF-subtracted $u$ image.

${ }^{\mathrm{d}}$ The upper limits of $u$ of components D and G are estimated by integrating the flux in the regions expected by the PSF-subtracted $i$ image, after subtracting PSFs. 
Table 3. EMISSION LINES

\begin{tabular}{|c|c|c|c|c|c|c|}
\hline \multirow[b]{2}{*}{ Element $(\AA)$} & \multicolumn{3}{|c|}{ Component A } & \multicolumn{3}{|c|}{ Component B } \\
\hline & $\lambda_{o b s}(\AA)$ & $\operatorname{FWHM}(\AA)$ & Redshift & $\lambda_{o b s}(\AA)$ & FWHM $(\AA)$ & Redshift \\
\hline $\mathrm{Al} \operatorname{III}(1857.40)$ & 4684.75 & 38.9 & $1.5222 \pm 0.003$ & 4690.14 & 42.4 & $1.5251 \pm 0.003$ \\
\hline Si III](1892.03) & 4775.94 & 36.0 & $1.5242 \pm 0.002$ & 4779.39 & 43.6 & $1.5261 \pm 0.003$ \\
\hline C III](1908.73) & 4815.97 & 48.5 & $1.5231 \pm 0.001$ & 4818.40 & 45.4 & $1.5244 \pm 0.002$ \\
\hline $\operatorname{Mg} \operatorname{II}(2798.75)$ & 7063.51 & 61.2 & $1.5238 \pm 0.001$ & 7063.01 & 60.8 & $1.5236 \pm 0.001$ \\
\hline
\end{tabular}


Table 4. ASTROMETRY FOR SDSS J0924+0219 AND NEARBY STARS

\begin{tabular}{ccccc}
\hline \hline Object & R.A. $(J 2000)^{\mathrm{a}}$ & Dec. $(J 2000)^{\mathrm{a}}$ & $\Delta$ R.A. $(\mathrm{sec})^{\mathrm{b}}$ & $\Delta$ Dec. $\left({ }^{\prime \prime}\right)^{\mathrm{b}}$ \\
\hline Component A & 092455.8293 & +021925.356 & +0.0108 & +0.847 \\
Component B & 092455.8327 & +021923.565 & +0.0142 & -0.944 \\
Component C & 092455.7659 & +021924.691 & -0.0549 & +0.182 \\
Component D & 092455.8653 & +021924.897 & +0.0468 & +0.388 \\
Component G & 092455.8185 & +021924.509 & 0.0000 & 0.000 \\
\hline Star a & 092457.7258 & +021844.566 & +1.9464 & -38.882 \\
Star b & 092455.1770 & +022025.660 & -0.6024 & +62.212 \\
Star c & 092451.6797 & +022019.861 & -3.9824 & +56.413 \\
\hline
\end{tabular}

a Astrometry is from Figure 3, based on the SDSS coordinates of stars a, b, and c. The errors of components A, B, C, D, and G (not including the absolute errors of stars a, b, and c) are 0.018, 0.042, 0.046, 0.123, and 0.085 pixel per coordinate, $(0.0012,0.0029,0.0032,0.0085$, and 0.0059 arcsecond per coordinate,) respectively.

${ }^{\mathrm{b}}$ Position relative to component $\mathrm{G}$ 
Table 5. OBSERVED AND PREDICTED POSITIONS OF THE LENSED COMPONENTS

\begin{tabular}{|c|c|c|c|c|c|c|}
\hline \multirow[b]{2}{*}{ component } & \multicolumn{3}{|c|}{ observed } & \multicolumn{3}{|c|}{ predicted } \\
\hline & $\Delta$ R.A..$\left.^{\prime \prime}\right)^{\mathrm{a}}$ & $\Delta$ Dec. $\left({ }^{\prime \prime}\right)^{\mathrm{a}}$ & $\operatorname{ratio}(* / \mathrm{A})^{\mathrm{b}}$ & $\Delta$ R.A..$\left.^{\prime \prime}\right)^{\mathrm{a}}$ & $\Delta$ Dec. $\left({ }^{\prime \prime}\right)^{\mathrm{a}}$ & $\operatorname{ratio}(* / A)^{b}$ \\
\hline Component A & +0.162 & +0.847 & 1.00 & +0.1688 & +0.8446 & 1.00 \\
\hline Component B & +0.213 & -0.944 & $0.44^{\mathrm{d}}$ & +0.2209 & -0.9289 & 0.38 \\
\hline Component C & -0.789 & +0.182 & $0.42^{\mathrm{d}}$ & -0.7548 & +0.1868 & 0.41 \\
\hline Component D & +0.702 & +0.388 & $0.09^{\mathrm{d}}$ & $\ldots$ & $\ldots$ & $\ldots$ \\
\hline Component $\mathrm{D}^{\prime c}$ & $\ldots$ & $\ldots$ & $\ldots$ & +0.6532 & +0.4962 & 0.85 \\
\hline
\end{tabular}

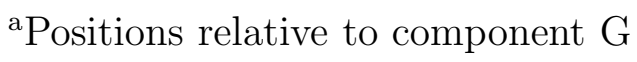

b * * " represents each component, A, B, C, D, and $\mathrm{D}^{\prime}$

'Predicted fourth lensed component

${ }^{\mathrm{d}}$ Mean ratio of all observed bands. 